\title{
Myelodysplastic Syndrome with Excess of Blasts - Long Term Partial Remission with Low Dose Prednisone, G-CSF and Epoetin
} Alfa

\author{
Anwarul Islam ${ }^{1}$ \\ ${ }^{1}$ Buffalo General Medical Center
}

May 29, 2021

\begin{abstract} G-CSF and Epoetin Alfa

Anwarul Islam, M.D., Ph.D., FRCPath, FACP

Clinical Associate Professor of Medicine

Division of Hematology/Oncology

Department of Medicine

Buffalo General Hospital, Room E 318

Buffalo, New York 14203
\end{abstract}

We have used low-dose prednisone, in conjunction with granulocyte, colony-stimulating factor (G-CSF) and erythropoietin, to treat an elderly patient with myelodysplastic syndrome (MDS) with an excess of blasts. Our findings indicate that such treatment is safe and may be effective in the long term survival of patients with high-risk MDS.

Myelodysplastic Syndrome with Excess of Blasts - Long Term Partial Remission with Low Dose Prednisone,

Tel: $716-859-2360$

E-mail: aislam@buffalo.edu

ABSTRACT

We have used low-dose prednisone, in conjunction with granulocyte, colony-stimulating factor (G-CSF) and erythropoietin, to treat an elderly patient with myelodysplastic syndrome (MDS) with an excess of blasts. Our findings indicate that such treatment is safe and may be effective in the long term survival of patients with high-risk MDS.

Key clinical message: Currently, most patients with high risk MDS are treated with 5-azacitidine or decitabine. These agents are not free of toxicity. Our treatment is safe, devoid of toxicity, and foster improved quality of life. There is reduced infection rate, red cell transfusion independence and little or no requirement of platelet infusion.

INTRODUCTION

Myelodysplastic syndromes (MDS) are a diverse group of hematological disorders that affect the blood and bone marrow (1). Different types of medications have been tried in MDS, however, no effective treatment has 
yet been established. Currently, most patients with high-risk MDS are treated with low-intensity regimens that are based on hypomethylating agents (HMAs) such as 5- azacitidine or decitabine (2). However, these chemotherapeutic agents are not free of toxicity. Across azacitidine and decitabine studies, approximately $50 \%$ of patients develop Common Toxicity Criteria (CTC) grade 3 or 4 cytopenias $(3,4)$, particularly during the first treatment cycle, necessitating intensive transfusion support, close monitoring, and placing patients at increased risk of febrile neutropenia episodes.

We have treated a newly diagnosed elderly patient with high-risk MDS with a combination of granulocytecolony-stimulating factor (G-CSF), low-dose prednisone and, recombinant erythropoietin (EPO). The rationale of such treatment was that this combination may induce differentiation of the immature, myeloid and erythroid precursor cells, inhibit proliferation of the blast cells, and potentially increase or stabilize the platelet count. Another logic behind this protocol was that this treatment may also improve the circulating neutrophil count, prevent serious infections, and avoid the undesirable effects of chemotherapy, thus improving the quality of life.

Our patient continued to demonstrate positive and satisfactory results 14 months after the therapy was started. The patient went into and remained in stable, continuous partial remission. His circulating neutrophil count increased and was generally within normal limits, while the hemoglobin level is maintained at $\sim 8 \mathrm{~g} / \mathrm{dL}$ without a need for blood transfusion. The platelet count has remained low $(\sim 40,000 / \mu \mathrm{L})$ but was stable and required no platelet transfusions. Occasional minor elevations of the neutrophil count (up to $15,000 / \mu \mathrm{L}$ ) were treated with diminished allocations of G-CSF. The peripheral blood smears continued to show a small number (1-4\%) of blasts and blast-like cells and morphologic features of myelodysplasia in all three cell lines. The patient's response was slow, to begin with but had been sustained over an extended period of time. Unfortunately, he contracted Covid-19 infection and died 10 days later, despite adequate treatment, including Covid-19 protocol, which involved dexamethasone, remdesivir and, convalescent plasma.

\section{CASE REPORT}

The patient, a 75-years-old white male with a past medical history significant for hypertension, chronic obstructive pulmonary disease, and hyperlipidemia, was brought to the emergency room (ER) with a history of syncopal attack. On physical examination, the patient was noted to be anemic, but he was not in acute distress. There was no jaundice, cyanosis, or edema. His abdomen was soft and non-tender. Bowel sounds were heard. The liver, spleen, and kidneys were not palpable. There was no palpable lymphadenopathy. Heart sounds S1 and S2 were identifiable, along with a soft ejection systolic murmur. The chest was clear to auscultation. His vital signs were stable -blood pressure 125/70 mm Hg, pulse $72 \mathrm{pm}$; respiration $18 \mathrm{pm}$, and temperature $97.6{ }^{*} \mathrm{~F}$.

Laboratory investigations revealed WBC $0.9 \times 10^{9} / \mathrm{L}$, hemoglobin $8.9 \mathrm{~g} / \mathrm{dL}$ with normal MCV, and $\mathrm{MCH}$, and a platelet count of $92 \times 10^{9} / \mathrm{L}$. A manual differential count of his peripheral blood smear revealed $47.0 \%$ neutrophils, $46.0 \%$ lymphocytes, $1 \%$ bands, $2 \%$ monocytes, $1 \%$ basophils, $1 \%$ blasts (Figure 1A), and $2 \%$ blast-like cells (Figure 1B). There were 10 nucleated RBCs per $100 \mathrm{WBC}$ (Figure 1C). The peripheral blood smear revealed hypogranularity and hypolobation (pseudo-Pelger-Huet anomaly) of neutrophils (Figure 1D), multinuclear (Figure 1E) and bizarre nuclear forms (Figure 1F). Red cells showed marked anisocytosis, poikilocytosis, dimorphic picture, presence of ovalocytes and macro-ovalocytes (Figure 2), and marked basophilic stippling (Figure 2 inset). The peripheral blood smear also displayed platelet anisocytosis and giant platelets (Figure 2). His serum iron level was normal at $70 \mu \mathrm{g} / \mathrm{dL}$ (normal range 45-182), the total iron binding capacity was normal at 374ug (normal range 261-478) and the percent saturation was slightly low at $19 \%$ (normal range 20-50). His reticulocyte count was raised at 7.5\% (normal range 0.5-1.5\%), The LDH was raised at 405 (normal range 105-210 U/l). His B12 (390 pg/ml) and folic acid (15.0 ng/ml) levels were also within normal limits. His complete metabolic profile was mostly normal, except for total bilirubin, which was slightly increased at $2.3 \mathrm{mg} / \mathrm{dL}$ (normal range $0.3-1.0 \mathrm{mg} / \mathrm{dL}$ ) and glucose was slightly raised at 151 $\mathrm{mg} / \mathrm{dL}$ (normal range 74-100). The routine urine analysis was negative.

Because of severe neutropenia, low hemoglobin level and abnormal cytologic findings in the peripheral 
blood smear, hematological malignancy was suspected and the patient was admitted for further evaluation, diagnosis and management. Soon after his admission to the hospital, the patient underwent a bone marrow examination, which revealed a slightly hypercellular ( $20 \%$ ) bone marrow (Figure 3 ) with the increased number of blast cells (12\%) (Figure 4) and marked dysplastic changes in all three cell lineages (Figure 5-7). There was marked basophilic stippling, but no ring sideroblasts were seen. The bone marrow biopsy section revealed focal clusters of immature myeloid precursor cells (Figure 8).

Flow cytometry studies of the peripheral blood sample revealed an increased blast cell population $2 \%$ of the events. The blast population was positive for dim CD45, CD34, CD117, and HLA-DR, while negative for CD13, CD33, CD14, CD15. The immunophenotype was compatible with a myeloblast. The granulocytes were slightly decreased and consisted mostly of mature-appearing granulocytes. No clonal B-cell or atypical T-cell population was detected.

Flow cytometry studies of the bone marrow aspirate sample revealed an increased population of cells that were positive for dim CD45, CD34, CD117, HLA-DR, and dim (CD10 (subset), while negative for CD13, CD33, CD14, CD15, B-and T-cell markers. No clonal B-cell or atypical T-cell population was detected (Figure 9).

Cytogenetic analysis of a 24-hour unstimulated bone marrow culture demonstrated an abnormal male karyotype. Of the 20 mitosis analyzed, one was normal and 19 showed an abnormal karyotype with an extra chromosome (trisomy 8) (Figure 10). FISH analysis was positive for trisomy 8 but negative for RUNX1/RUNX1T1, CBFB/MYTH11, BCR/ABL, MECOM, KMT2A rearrangement, and MONOSOMY 5 (DELETION OF 5Q), MONOSOMY 7 (DELETION OF 7Q), MONOSOMY 20 (DELETION OF 20Q), ATM (DELETION OF TP53) or 13Q14 deletion. Molecular studies for FLT3, IDH1, IDH2 and TP53 were negative. The patient was diagnosed to have high-risk MDS, which was determined by the age, cytopenias, percentage of blasts in the marrow and abnormal cytogenetic profile (trisomy 8).

Because of the patient's age (75 years) and a diagnosis of MDS with excess of blasts (high risk), the patient was referred to hospice care. However, the patient's family declined this option and requested a treatment that did not involve chemotherapy. At this point, the patient was started on low-dose prednisone $20 \mathrm{mg}$ orally daily, G-CSF (filgrastim) 300ug subcutaneously three times a week (Monday, Wednesday and Friday) and epoetin (procrit) 30,000 units subcutaneously once a week. The patient did not receive any other cytokines or chemotherapy. Prednisone was gradually tapered and was reduced to $5 \mathrm{mg}$ orally daily for the last 6 months, G-CSF was reduced to $300 \mu \mathrm{g}$ once a week; and epoetin was reduced to 30,000 units once every two weeks for the last four months.

Within two weeks of the start of the cited treatment, his circulating neutrophil count began to increase. The hemoglobin concentration and platelet count remained low $(\mathrm{Hb} \sim 8 \mathrm{~g} / \mathrm{dL}$, platelet $\sim 40-60,000 / \mu \mathrm{L})$ but stable and did not require RBC or platelet transfusion. The peripheral blood smear continued to show a small number $(2-4 \%)$ of blast and blast-like cells but no overt signs of leukemic transformation. As stated earlier the patient contracted Covid-19 infection and expired soon thereafter.

\section{DISCUSSION}

The ideal therapeutic approach for older (age [?] 65 years) patients with MDS, particularly high-risk groups of patients, is not known. Although allogeneic hematopoietic stem-cell transplantation can induce long-term remission in patients with MDS (5-8), such therapy is not applicable for most patients, since the median age at diagnosis usually exceeds 70 years (9) as is in the case under discussion. Challenges facing current standard-of-care therapies for high-risk MDS include poor overall survival, limited duration of response, and singular mechanistic action $(3,10,11)$. Responses achieved with currently available treatment are often less than one year of duration (11) and these therapies focus on either decreasing leukemic proliferation or limiting immune activation $(10,12)$. Many high-risk MDS patients require repeated blood transfusions and are often at risk for serious infections and hospitalization.

In general high-risk elderly patients are treated with demethylating agents such as 5-azacitidine or decitabine 
since the publication of an article that demonstrated treatment with demethylating agent results in superior remission rates, as compared with supportive care, and in some cases a delay in blastic transformation (13). In a recent randomized study that compared azacitidine treatment with conventional care in patients with high-risk disease, the median survival was 24.5 months in the azacitidine group, as compared with 15.0 months in the conventional care group (3). However, treatment for elderly patients with high-risk MDS with demethylating agents is not free of toxicity and treatment-related mortality can be quite high $(3,4,14)$. Furthermore, once a drug fails in one of these patients, further treatment options are limited and median survival is less than six months (15).

MDS has been considered a malignant condition and consequently treatment of this condition has been chemotherapy, except for a few studies where G-CSF and erythropoietin were tried in patients with low risk disease and minimal transfusion-dependence $(16,17)$. We took a different approach, believing that perhaps this condition is a manifestation of an underlying disorder (18) rather than a malignant disorder. Accordingly, we treated our patient not with chemotherapy but with a combination of prednisone, and myeloid and erythroid-stimulating factors such as G-CSF and EPO. We believe this is the first-reported high-risk MDS patient who was treated in this combination. It achieved excellent results. We believe this observation merits further evaluation and a prospective trial. However, as we have reported earlier (18), the diagnosis of MDS is not uniform and considerable variations exist among the MDS patients as well as diagnosticians. This disease is often misclassified as evidenced by the fact that over $50 \%$ of patients in certain registries were considered "MDS - unclassifiable" $(9,19)$. When bone marrow biopsies are not studied in parallel with bone marrow aspiration during the investigation and diagnosis of MDS, the abnormal localization of blast cells may be missed and as a result, high-grade MDS may be misdiagnosed as low or intermediate-risk thus jeopardizing the correct stratification of this disease and thereby influencing the prognostic outcome. As a result, one has to be mindful of comparing apples and oranges in the setting of the same or similar treatment.

\section{References}

1.Snowden J A, O 'Connell S, Hawkins J, et al. Haematological cancers: improving outcomes. A summary of updated NICE service guidance in relation to Specialist Integrated Haematological Malignancy Diagnostic Services (SIHMDS) J Clin Pathol 2017;70:461-468 doi: 10.1136/jclinpath-2016-204029

2.Favor M G, Griffiths E A, Thota S. Current state of myelodysplastic syndromes: standard treatment practices and therapeutic advances. J Clin Pathways 2019;5:43-47

3.Fenaux P, Mufti G J, Hellstrom-Lindberg E, et al. International Vidaza High-Ris MDS survival Study Group. Efficacy of azacitidine compared with that of conventional care regimens in the treatment of higherrisk, myelodysplastic syndrome: a randomized, open-label, phase III study. Lancet Oncol. 2009;10: 223-232.

4. Lubbert M, Suciu S, Baila L, et al. Low-dose decitabine versus best supportive care in elderly patients with intermediate- or high-risk myelodysplastic syndrome (MDS) ineligible for intensive chemotherapy: final results of the randomized phase III study of the European Organization for Research and Treatment of Cancer Leukemia Group and the German MDS Study Group. J Clin Oncol. 2011; 29: 1987-1996

5. Chang C, Storer B E, Scott B L, et al., Hematopoietic cell transplantation in patients with myelodysplastic syndromes or acute myeloid leukemia arising from myelodysplastic syndrome: similar outcomes in patients with de novo disease and disease following prior therapy or antecedent hematologic disorders, Blood 2007;110:1379-1387.

6. Warlick E D, Cioc A, Defore T, et al. , allogeneic stem cell transplantation for adults with myelodysplastic syndromes: importance of breed transplant disease burden. Biol Blood Marrow Transplant 2009;15:30-38.

7. Alessandrino E P, Porta M G, Malcovati L, et al. , on behalf of Gruppo Italiano Trapianto di Midollo Osseo (GITMO). Optimal timing of allogeneic hematopoietic stem cell transplantation in patients with myelodysplastic syndrome. Am J Hematol 2013.

8. Platzbecker U, Schetelig J, Finke J, et al., German MDS study; Cooperative Transplant Study Ggroup; 
Fred Hutchinson Cancer Research Center: group Francophone des Myelodysplasies. Allogeneic hematopoietic cell transplantation in patients age 60-70 years with de novo high-risk myelodysplastic syndrome or secondary acute myelogenous leukemia: comparison with patients lacking donors who received azacitidine. Biol Blood Marrow Transplant. 2012; 18 1415-1421.

9. Rollison D E, Howlader N, Smith, M T, et el. Epidemiology of myelodysplastic syndromes and chronic myeloproliferative disorders in the United States, 2001-2004, using data from NAACCR and SEER programs. Blood. 2008;112:45-52.

10. Kadia T M, Jabbour E, Kantarjian H. Failure of hypomethylating agent- based therapy in myelodysplastic syndromes. Semin Oncol. 2011; 38: $682-692$.

11. Platzbecker U. treatment of MDS. Blood. 2019;133:1096-1107

12. Kordasti S Y, Ingram W, Hayden J, et al. CD4+CD25high Foxp3+regulatory cells in myelodysplastic syndrome (MDS) Blood. 2007;110:847-850.

13. Silverman L R, Demakos E P, Peterson B L, et al. Randomized controlled trial of azacitidine in patients with the myelodysplastic syndrome: a study of the Cancer and Leukemia Group B. J Clin Oncol 2002:20:2429-2440.

14. Gao C, Wang J, Li Y, et al. Incidence and risk of hematologic toxicities with hypomethylating agents in the treatment of myelodysplastic syndromes and acute myeloid leukemia. A systematic review and metaanalysis. Medicine 2018; 97: 1-13

15. Sekeres M A, and Cutler C. How we treat higher- risk myelodysplastic syndromes. Blood;123: 829-836

16. Hussain I S. Myelodysplastic syndromes in the elderly: role of growth factors in management. Leukemia Research. 1996; 20: 203-219.

17. Jadersten M, Malcovati L, Dybedal I, et al. Erythropoietin, and granulocyte-colony stimulating factor treatment associated with improved survival in myelodysplastic syndrome. J Clin Oncol. 2008; 26: 36073613 .

18. Islam A. Myelodysplastic Syndromes: A Diagnostic and Therapeutic Conundrum. Ann Hematol Oncol. 2020; 7(8): 1318 .

19. Cogle C R, Craig B M, Rollison D E, et.al. Incidence of the myelodysplastic syndromes using a novel claims-based algorithm: highly number of uncaptured cases by cancer registries. Blood. 2011; 117:7121-7125.

Legends:

Figure 1 (A) A myeloblast (note a prominent nucleolus), (B) a blast-like cell (a similar cell as A but note absence of nucleolus, (C) two nucleated red blood cells (D) bilobed and 'spectacle' forms (note hypogranulation), (E) multi nuclear and (F) bizarre forms.

Figure 2. A peripheral blood smear showing dimorphic blood picture along with anisocytosis, poikilocytosis and presence of ovalocytes, macro-ovalocytes. Note platelet anisocytosis and giant platelets. The inset (lower right hand corner) shows a red blood cell with basophilic stippling.

Figure 3. Bone marrow biopsy section showing slightly hypercellular marrow.

Figure 4. Bone marrow aspirate smear showing a myeloblast. Note prominent nucleoli.

Figure 5. Bone marrow aspirate smear showing dysplastic changes in erythroid precursors. (A)- intercytoplasmic bridging, (B) - nuclear budding, (C)-nuclear cytoplasmic maturation asynchrony.

Figure 6. Bone marrow aspirate smear showing dysplastic changes in granuloid precursors. A- pseudo Pelger-Huet anomaly, B- bizarre nuclei, C- a giant metamyelocyte. 
Figure 7. Dysmegakaryopoiesis: multi nuclear megakaryocytes, top row, bone marrow aspirate smear. Bottom row: mono, bi and multi-nucleated megakaryocytes-bone marrow biopsy section.

Figure 8. Bone marrow biopsy section demonstrating focal clusters of immature myeloid precursors (blast cells) which can be easily identified by the presence of nucleolus and nucleoli. Two cells in mitosis (arrows) can be seen.

Figure 9. Flow cytometry results of bone marrow aspirate obtained at diagnosis: cluster analysis of blasts using CD45/SSC. Pink: blasts; green: lymphocytes; blue: granulocytes; red: erythroid precursors and debris and orange; monocytes.

Figure 10. Fluorescent in situ hybridization of bone marrow cells identify an abnormal karyotype with an extra chromosome 8 (trisomy 8 ).
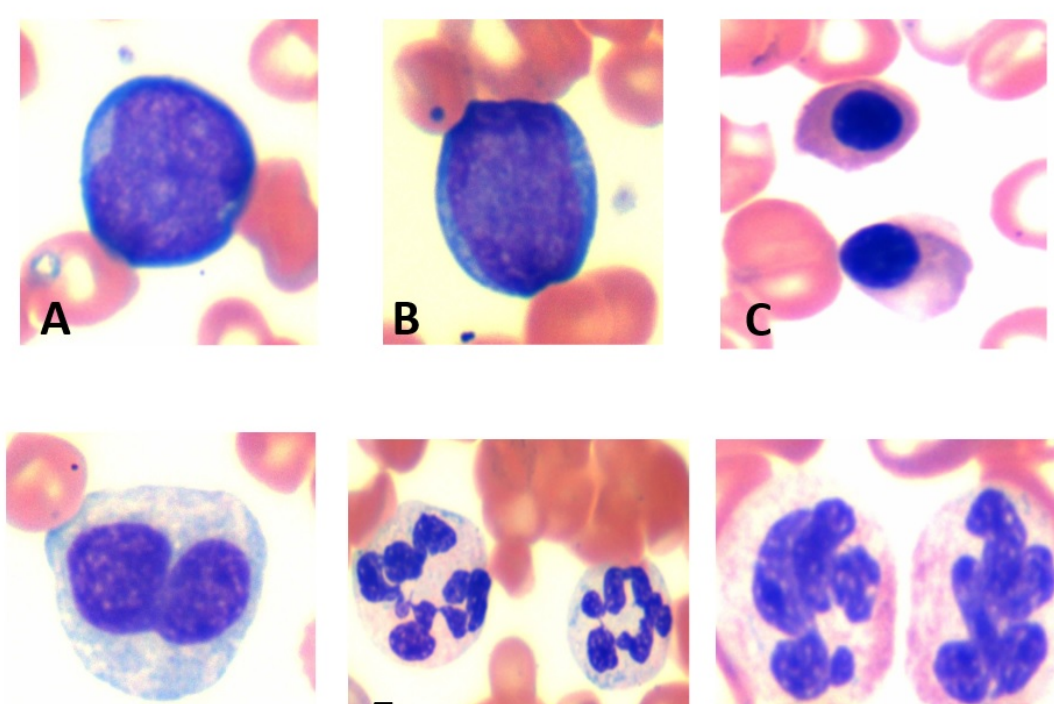

D
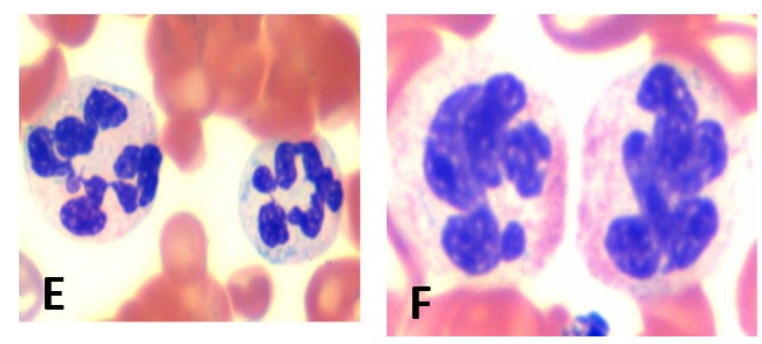

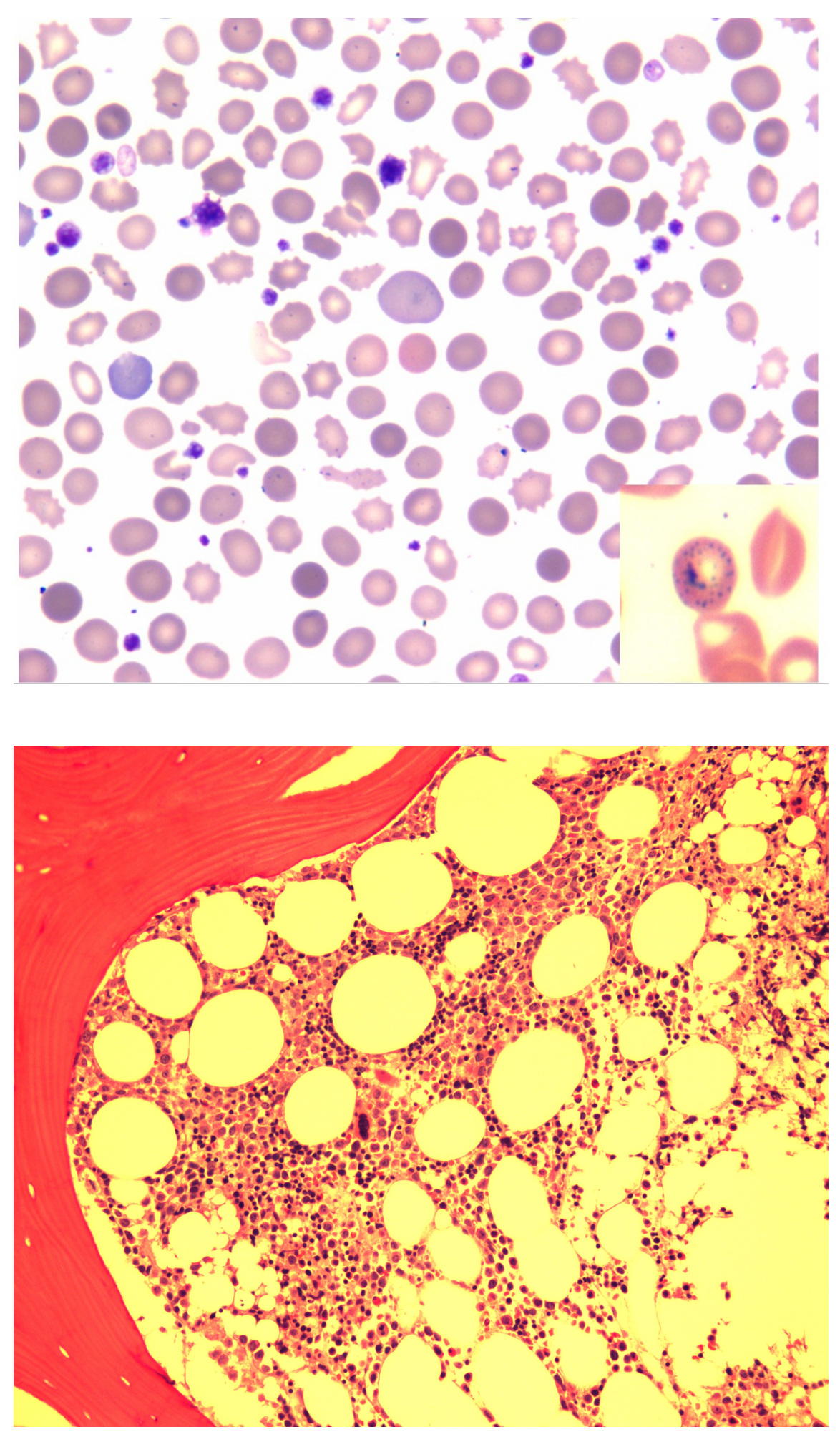

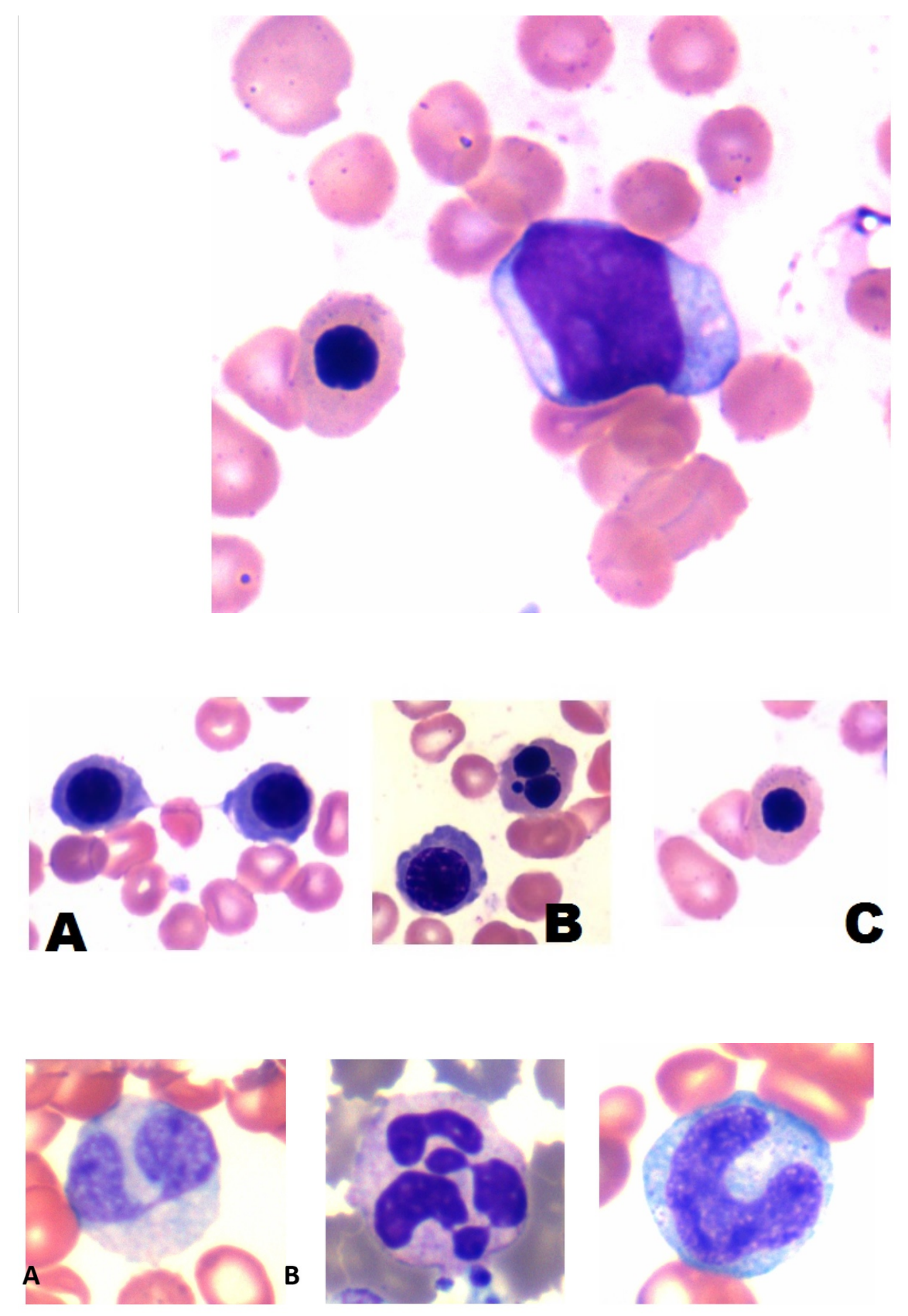


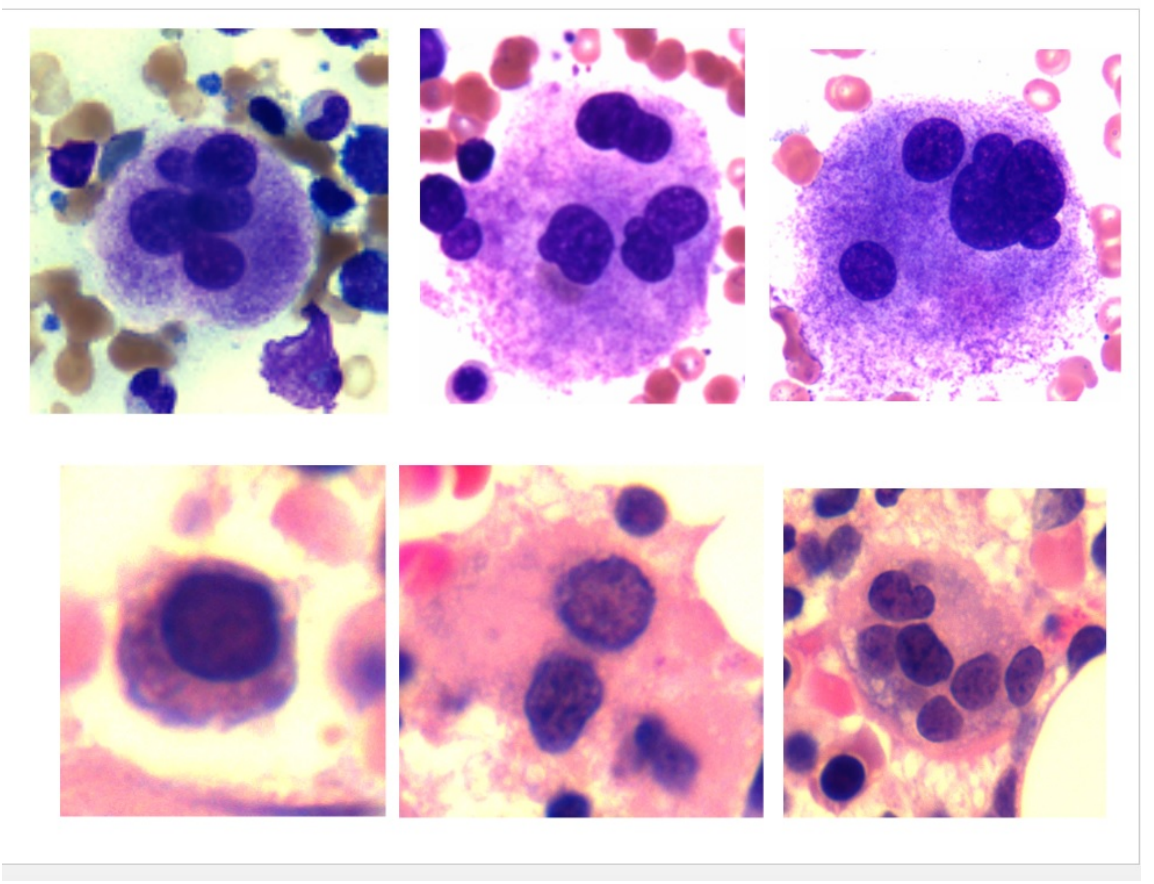

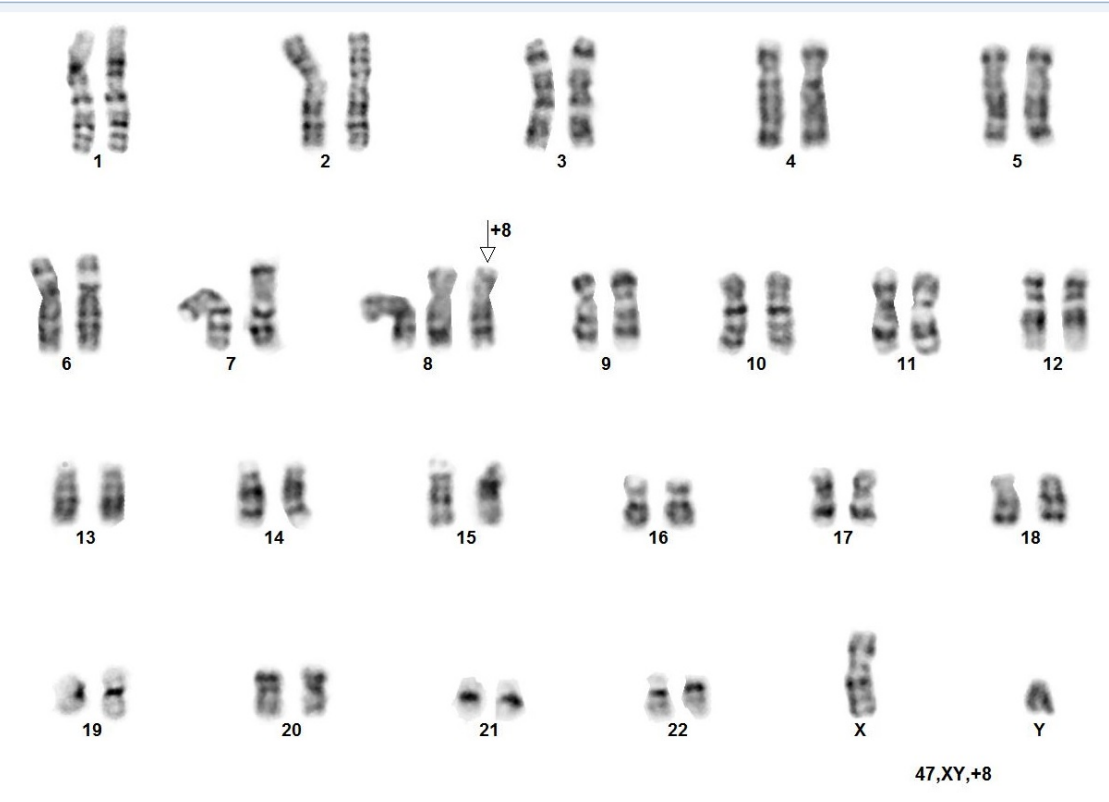



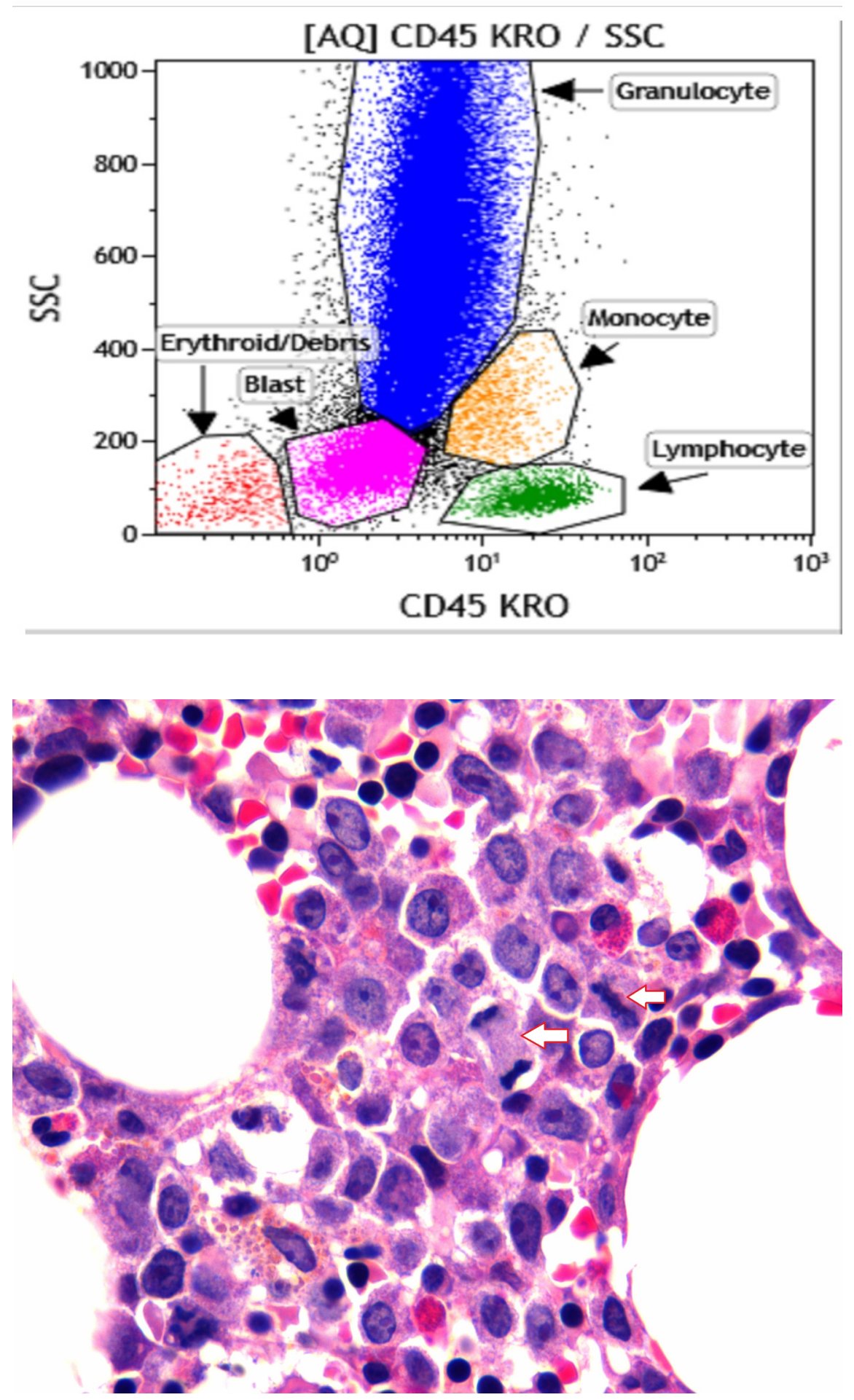